\title{
Electricity market and its risk management in Nigeria
}

\author{
Achudume Celestine", Chukwuma Raphael Nwozo \\ Department of Mathematics, University of Ibadan, Oyo State, Nigeria
}

Email address:

celeachudume@gmail.com (A. Celestine)

\section{To cite this article:}

Achudume Celestine, Chukwuma Raphael Nwozo. Electricity Market and Its Risk Management in Nigeria. Applied and Computational Mathematics. Vol. 3, No. 5, 2014, pp. 256-261. doi: 10.11648/j.acm.20140305.20

\begin{abstract}
This paper is on the development of adequate mathematical model of electricity price via Fourier series. Fourier series is the representation of a function $f(x)$ as an infinite series in sine and cosine terms. Our choice of Fourier series model for electricity price is as result of its volatility, fluctuation trends of hydro flow and poor market designs and we use activelytraded natural gas to hedge against electricity price in Nigeria. The natural gas prices are volatile but do not have a clear seasonal pattern, thus eliminating natural gas price volatility through hedging substantially reduce the electricity price, this development of logical mathematical frame work in the form of hedging tools assures an investor of his or her safety in the power sector.
\end{abstract}

Keywords: Fourier Series, Electricity Market, Seasonality, Hedging Risk

\section{Introduction}

In this paper we consider investors who have the intention to expand the electricity generation capacity in Nigeria. The newly liberalized electricity markets in Nigeria where, electricity is traded like every other commodity as attracted the attention of both the foreign and local investors into the power sector. The motivation for this work are those of [2] who observe the detection of market-power abuse and price manipulation while an improvement on this fact made by [4] with the hypothesis that a forward-contract seller is relatively less risk averse than buyer, subsequently, In [24], [25], [26] discovered the presence of relatively large premium corroborates of the electricity price, just to mention a few. In this paper we consider logical mathematical frame work via Fourier series model for the pricing of electricity, given as $f(x)=\frac{1}{2} a_{0}+\sum_{n=0}^{\infty} a_{n}+\cos n x+b_{n} \sin n x$ where the coefficients $a_{0}, a_{n}, b_{n}$, denotes the drift term and volatility respectively and $f(x)$ denotes the i.i.d. random variable and subsequently show that given a contingent claim ' $S$ ', such that $\mathbb{E} \gamma^{*}\left(S^{2}\right)<\infty$ is called attainable if there exists at least one tame self-financing trading strategy $\emptyset$ such that

$V_{(T)}^{\emptyset}=S$ and we show that electricity market is complete (it can be hedge)

\section{Mathematical Formulations}

(Dynamics of electricity pricing)

\section{Theorem 1}

Given $f(x)=\frac{1}{2} a_{0}+\sum_{n=0}^{2} a_{n} \cos n x+b_{n} \sin n x$ where the coefficients $a_{0}, a_{n}, b_{n}$

Denotes the drift and volatility respectively and $f(x)$ denotes an i.id random variable where,

$W=\left\{W_{t}, t \in[0, T]\right\}$ is a Brownian motion defined in a complete probability space $(\Omega, \mathcal{F}, \mathbb{P})$. We will denote by $\left\{f_{t}: t \in[0, T]\right\}$ the filtration generated by the Brownian motion and completed by the $\mathbb{p}-$ null sets

$$
f(x)=\frac{1}{2} a_{0}+\sum_{n=0}^{2} a_{n} \cos n x+b_{n} \cos n x x \in[0, \pi]
$$

Where $a_{0}=\pi$ the drift rate and $a_{n}, b_{n}$ the volatility including all the economic factors; where $a_{n}=$ $\frac{2}{\pi n^{2}}(\cos n \pi-1), \quad b_{n}=\frac{-2 \cos n \pi}{n}$;

And

$f_{p^{L}}(x)=\left(\frac{\pi}{2}\right)+\sum_{n=0}^{2} \frac{2}{\pi n^{2}}(\cos n \pi-1) 1+e^{-\frac{n^{2}}{2} t}+\left(\frac{-2 \cos n \pi}{n}\right) e^{\frac{n^{2} t}{2}}$

Proof:

$a_{0=} 1 / \pi \int_{0}^{\pi} 2 x d x$ where $f(x)=2 \pi$, we consider only $0 \leq x \leq \pi, 0 \leq t \leq \pi$, and $0 \leq n \leq 2$

$$
a_{0}=\left[\frac{1}{\pi} \cdot \frac{2^{x^{2}}}{2}\right]_{0}^{\pi}=\frac{2 \pi^{2}}{2 \pi}=\frac{\pi^{2}}{\pi}=\pi
$$

$a_{n}=\frac{1}{\pi} \int_{0}^{\pi} f(x) \cos n x d x$ 


$$
\begin{aligned}
=\frac{1}{\pi} \int_{0}^{\pi} 2 x \cos n x d x & =\frac{2}{\pi} \int_{0}^{\pi} x \cos n x d x \\
U d v & =U v \int V d u
\end{aligned}
$$

Let $u=x$ and $d v=\operatorname{cosn} x$

$d u=d x$ and $V=\frac{\operatorname{sinn} x}{n}$

$\frac{2}{\pi}\left[\left.\frac{x \sin n x}{n}\right|_{0} ^{\pi}-\int_{0}^{\pi} \frac{\sin n x}{n} d x\right]=\frac{2}{\pi}\left[\left.\frac{x \sin n x}{n}\right|_{0} ^{\pi}+\left.\frac{\cos n x}{n^{2}}\right|_{0} ^{\pi}\right]=\left[\left.\frac{2 \cos n x}{\pi n^{2}}\right|_{0} ^{\pi}\right]$ $\left(\left.\operatorname{since} \frac{x \sin n x}{n}\right|_{0} ^{\pi}=0\right)$

$$
\begin{aligned}
& \frac{2}{\pi n^{2}}(\cos n \pi-\cos n 0)=\frac{2}{\pi n^{2}}(\cos n \pi-1)(\operatorname{since} \cos n 0=1) \\
& \therefore a_{n}=\frac{2}{\pi n^{2}}(\cos n \pi-1)=\frac{-4}{\pi n^{2}} \text { for } n \text { odd }, 0 \text { for } n \text { even. }
\end{aligned}
$$

For $b_{n}$

$$
b_{n}=\frac{1}{\pi} \int_{0}^{\pi} f(x) \sin n x d x
$$

$b_{n}=\frac{1}{\pi} \int_{0}^{\pi} 2 x \sin n x d x \Rightarrow b_{n}=\frac{2}{\pi} \int_{0}^{\pi} x \operatorname{sinn} x d x$, by $u d v=$ $u v-\int_{0}^{t} v d u$

Let $x=u d x=d u$ and $d v=\operatorname{sinn} x$

$$
v=\frac{-\cos n x}{n}, \Rightarrow \frac{2}{\pi}\left[\left.\frac{-x \cos n x}{n}\right|_{0} ^{\pi}+\frac{1}{n} \int_{0}^{\pi} \cos n x d x\right]=\frac{2}{\pi}\left[\left.\frac{-x \cos n x}{n}\right|_{0} ^{\pi}+\left.\frac{\operatorname{sinn} x}{n^{2}}\right|_{0} ^{\pi}\right] \frac{-2}{\pi n}\left[\left.x \cos n x\right|_{0} ^{\pi}\right] \quad\left(\operatorname{since} \frac{1}{n^{2}}\left[\left.\sin n x\right|_{0} ^{\pi}\right]\right)=0 \Rightarrow
$$

$\frac{-2}{\pi n}[\pi \cos n \pi-0 . \cos n 0]=\frac{-2}{\pi n}[\pi \cos n-0]=\frac{-2}{\pi n}(\pi \cos n \pi)=\frac{-2 \cos n \pi}{n} \therefore \Rightarrow b_{n}=\frac{-2 \cos n \pi}{n}=\frac{2}{n}$ for $n$ odd $\frac{-2}{n}$ for $k$ even

Substituting for $a_{0}, a_{n}$, and $b_{n}$ into $\quad f(x)=\frac{1}{2} a_{0}+$ $\sum_{n=1}^{2} a_{n} \cos n x+b_{n} \cos n x$

$$
f(x)=\frac{\pi}{2}+\sum_{n=1}^{2} \frac{2}{\pi n^{2}}(\cos n \pi-1) \cos n x+\left(\frac{-2 \cos n \pi}{n}\right) \sin n x
$$

Thus, we solve for $\operatorname{cosnw}(t)$ and $\operatorname{sinnw}(t)$ stochastically finding the mathematical expectation of

$$
\mathbb{E}(\operatorname{cosn} w(t)) \text { and } \mathbb{E}(\operatorname{sinnw}(t))
$$

$\mathbb{E}(\operatorname{cosn} w(t))$

Let $U=\cos n x$ and $\frac{\partial u}{\partial x}=-n \operatorname{sinn} x, \frac{\partial^{2} u}{\partial x^{2}}=-n^{2} \cos n x$, $\frac{\partial u}{\partial t}=0$

Using Itô lemma, we have,

$$
\begin{gathered}
d u(t, x(t))=\left(\frac{\partial u}{\partial t}+a \frac{\partial u}{\partial t}+\frac{b^{2}}{2} \frac{\partial^{2} u}{\partial x^{2}}\right) d t+\frac{\partial u}{\partial x} d w(t) \\
U(t, x(t))=U(0, x(0))+\int_{0}^{t}\left(\frac{\partial u}{\partial s}+\frac{1}{2} \frac{\partial^{2} u}{\partial x^{2}}\right) d s+\int_{0}^{t} \frac{\partial u}{\partial x} d w(s) \\
\cos n x(t)=\cos n x(0)+\int_{0}^{t} \frac{-n^{2}}{2} \cos n x(s) d s+\int_{0}^{t}-n \operatorname{sinn} x(s) d w(s) \\
\cos n x(t)=1+\int_{0}^{t} \frac{-n^{2}}{2} \cos n w(s) d s+\int_{0}^{t}-n \operatorname{sinn} x(s) d w(s) \\
\mathbb{E}(\cos n x(t))=\mathbb{E}(1)-\frac{1}{2} \int_{0}^{t} \mathbb{E}\left(n^{2} \cos n x(s)\right) d s-\int_{0}^{t} \mathbb{E}(n \operatorname{sinn} x(s)) d w(s) \\
\mathbb{E}(\cos n x(t))=1-\frac{1}{2} \int_{0}^{t} \mathbb{E}\left(n^{2} \cos n x(s)\right) d s \\
\mathbb{E}(\cos n x(t))=1-\frac{n^{2}}{2} \int_{0}^{t} \mathbb{E}(\cos n x(s)) d s \\
L(t)=1-\frac{n^{2}}{2} \int_{0}^{t} L(s) d s
\end{gathered}
$$

$$
\begin{gathered}
L(t)=1-\frac{n^{2}}{2} L(t), \quad L(0)=1 \\
\frac{d L(t)}{d t}=\frac{-n^{2}}{2} L(t) \\
\int \frac{d L(t)}{L t}=\int_{0}^{t} \frac{-n^{2}}{2} d s \\
\ln L(t)=\frac{-n^{2} t}{2}+c \\
\ln (1)=0+c \\
0=c \ln L(0)=0+c
\end{gathered}
$$

Then we have, $\ln L(t)=\frac{-n^{2} t}{2}$

$$
L(t)=e^{-\frac{n^{2} t}{2}}
$$

Thus,

$$
\operatorname{cosnw}(t)=e^{\frac{-n^{2} t}{2}}
$$

Synonymously,

We have that

$$
\begin{gathered}
\operatorname{sinnw}(\mathrm{t})=\mathrm{e}^{-\mathrm{inw}(\mathrm{t})} \\
U(t, X(t))=e^{-i n w(t)}=e^{-i n x} \\
\frac{\partial \mathrm{u}}{\partial \mathrm{t}}=0, \frac{\partial \mathrm{u}}{\partial \mathrm{x}}=-i n \mathrm{e}^{-\mathrm{inx}} ; \frac{\partial^{2} \mathrm{u}}{\partial \mathrm{x}^{2}}=\mathrm{n}^{2} \mathrm{e}^{-\mathrm{inx}}
\end{gathered}
$$

Substituting into Itô lemma below $d u(t, x(t))=$ $\left(\frac{\partial u}{\partial t}+a \frac{\partial u}{\partial t}+\frac{b^{2}}{2} \frac{\partial^{2} u}{\partial x^{2}}\right) d t+\frac{\partial u}{\partial x} d w(t)$ we have 


$$
\begin{gathered}
\operatorname{sinnw}(t)=0+\int_{0}^{t} 0+0+\frac{n^{2}}{2} e^{-i n x} d s+n \int_{0}^{t}-i n e^{-i n w(s)} d w(s) \\
\operatorname{sinnw}(t)=0+\int_{0}^{t} 0+0+\frac{n^{2}}{2} e^{-i n w(s)} d s+n \int_{0}^{t}-i n e^{-i n w(s)} d w(s) \\
\operatorname{sinnw}(t)=\frac{n^{2}}{2} \int_{0}^{t} e^{-(n w(s))} d s-n i \int_{0}^{t} \mathbb{E}\left(e^{-i n w(s)}\right) d w(s)=\frac{n^{2}}{2} \int_{0}^{t} e^{\frac{n^{2}}{2} s} d s=\frac{n^{2}}{2} \cdot \frac{2}{n^{2}} \cdot e^{\frac{n^{2}}{2} t}=e^{\frac{n^{2}}{2} t}
\end{gathered}
$$

similarly,

$\mathbb{E}(\operatorname{cosn} w(t))$

We also recall that $\operatorname{cosn} w(t)=e^{i n w(t)}$

Let $\mathrm{U}=e^{i n x}, \frac{\partial u}{\partial x}=i n e^{i n x}, \frac{\partial^{2} u}{\partial x^{2}}=-n^{2} e^{i n x}, \frac{\partial u}{\partial t}=0$

$$
\begin{gathered}
\operatorname{cosnw}(t)=1+\int_{0}^{t} 0+0-n^{2} e^{i n w(s)} d s \\
+\int_{0}^{t} \operatorname{ine} e^{i n w(s)} d w(s)
\end{gathered}
$$

$$
\operatorname{cosnw}(t)=1-\int_{0}^{t} n^{2} e^{i n w(s)} d s+\int_{0}^{t} \operatorname{in} e^{i n w(s)} d w(s)
$$

$\mathbb{E}(\operatorname{cosn} w(t))=1-\int_{0}^{t} \frac{n^{2}}{2} \mathbb{E}\left(e^{i n w(s)}\right) d s+\int_{0}^{t} \operatorname{in} \mathbb{E}\left(e^{i n w(s)}\right) d w(s)$

$=1+\frac{n^{2}}{2} \int_{0}^{t} E\left(e^{i n w(s)}\right) d s+\int_{0}^{t} i n \mathbb{E}\left(e^{i n w(s)}\right) d w(s)$

$=1-\frac{n^{2}}{2} \int_{0}^{t} e^{-\frac{n^{2}}{2} t} d s$

$=1+\frac{n^{2}}{2} \cdot \frac{2}{n^{2}} e^{-\frac{n^{2}}{2} t}$

$=1+e^{-\frac{n^{2}}{2} t}$

Thus combining (6), (7), (8) i.e.

$\operatorname{sinn} w(t)+\operatorname{cosn} w(t)$, we have

$$
1+e^{\frac{n^{2}}{2} t}+e^{-\frac{n^{2}}{2} t}
$$

But note that $\operatorname{cosn} w(t)+\operatorname{sinn} w(t)$

$$
e^{\frac{n^{2} t}{2}+} e^{\frac{-n^{2} t}{2}}=1-x+\frac{x^{2}}{2 !}-\frac{x^{3}}{3 !}-\frac{x^{7}}{7 !}
$$

if $x=\frac{n^{2} t}{2}$,Thus Substituting both (2), (3), (4)-(10) into (11), we have

$$
\begin{gathered}
f_{p^{L}}(x)=\left(\frac{\pi}{2}\right)+\sum_{n=0}^{2} \frac{2}{\pi n^{2}}(\cos n \pi-1) 1+e^{-\frac{n^{2}}{2} t}+ \\
\left(\frac{-2 \cos n \pi}{n}\right) e^{\frac{n^{2} t}{2}}
\end{gathered}
$$

Where $e^{\frac{-n^{2} t}{2}}=1+\frac{x^{2}}{2 !}+\frac{x^{2}}{4 !}+\frac{x^{6}}{6 !}$ And $e^{\frac{-n^{2} t}{2}}=1-\frac{x^{2}}{2 !}-$ $\frac{x^{3}}{3 !}-\frac{x^{7}}{7 !}$

\section{Simulation}

From (11),

$f_{p^{L}}(x)=\left(\frac{\pi}{2}\right)+\sum_{n=0}^{2} \frac{2}{\pi n^{2}}(\cos n \pi-1) 1+e^{-\frac{n^{2}}{2} t}+$ $\left(\frac{-2 \cos n \pi}{n}\right) e^{\frac{n^{2} t}{2}}$ Taken values when $0 \leq n \leq 2$

And $0 \leq x \leq \pi$ and $0 \leq t \leq \pi$

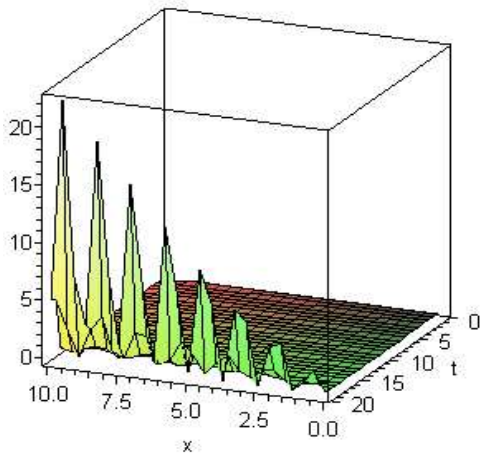

Figure 1.

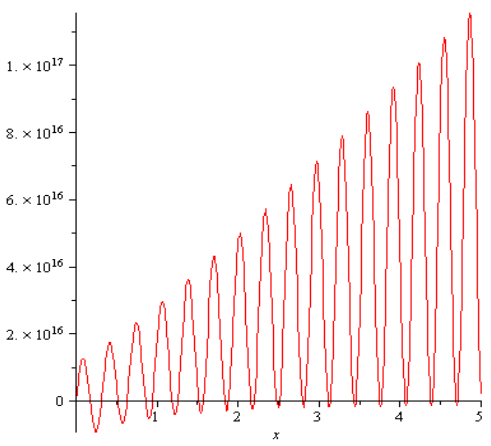

Figure 2.

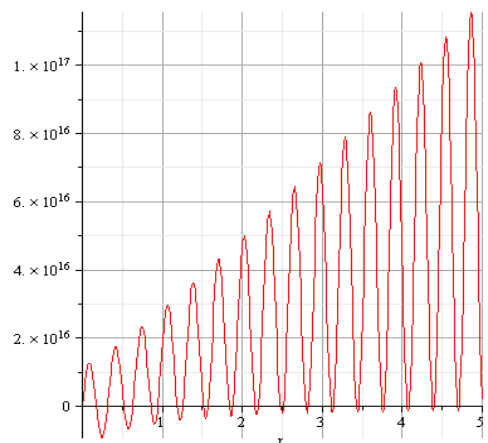

Figure 3. 


\section{Methodology}

\section{(Hedging tools)}

We consider a market consisting one stock (risky asset) as the prices of our natural gas and one bond (riskless asset). The prices process of the risky asset is assumed to be of the form $S_{t}=S_{0} e^{N_{t}}, \quad t \in[0, T]$ with

$$
N_{t}=\int_{0}^{t}\left(\mu_{s}-\frac{\sigma^{2}}{2}\right) d s+\int_{0}^{t} \sigma_{s} d w(s)
$$

Where, $W=\left\{W_{t}, t \in[0, T]\right\}$ is a Brownian motion defined in a complete probability $\operatorname{space}(\Omega, \mathcal{F}, \mathbb{P})$. We will denote by $\left\{f_{t}: t \in[0, T]\right\}$ the filtration generated by the Brownian motion and completed by the $\mathbb{p}-$ null sets. The mean rate of return $\mu_{t}$ and the volatility process $\sigma_{t}$ are measurable and adapted satisfying the following integrability conditions

$$
\int_{0}^{t}\left|\mu_{t}\right| d t<\infty, \quad \int_{0}^{t} \sigma_{t}^{2} d t<\infty \text { a.s. }
$$

By Itô's formula, we obtain that $S_{t}$ satisfies the linear stochastic differential equation:

$$
d s_{t}=\mu_{t} s_{t} d t+\sigma_{t} s_{t} d w_{t}
$$

The price of the bond $\beta_{t}, t \in[0, T]$, evolves according to the differential equation $d \beta_{t}=r_{t} \beta_{t} d t, \beta_{0}=1$, where the interest rate process is a non-negative measurable and adapted process satisfying the integrability condition $\int_{0}^{t} r_{t} d t<\infty$, a.s i.e.

$$
\beta_{t}=\exp \left(\int_{0}^{t} r_{s} d s\right)
$$

Imagine an investor who starts with some initial endowment $x \geq 0$ and invests in the assets describe above. Let $\alpha_{t}$ be the number of non-risky assets and $\beta_{t}$ the number of stocks owned by the investor at time $t$. The couple $\emptyset_{t}=\left(\alpha_{t}, \beta_{t}\right), t \in[0, T]$, is called a portfolio or trading strategy, and we assume that $\alpha_{t}$ and $\beta_{t}$ are measurable and adapted processes such that

$\int_{0}^{t}\left|\beta_{t} \mu_{t}\right| d t<\infty, \int_{0}^{t} \beta_{t}^{2} \sigma_{t}^{2} d t<\infty, \int_{0}^{t}\left|\alpha_{t}\right| r_{t} d t<\infty$ a.s.

Then $x=\alpha_{0}+\beta_{0} S_{0}$, and the investor's wealth at time $t$ (also called the value of the portfolio) is

$$
V_{t}(\varnothing)=\alpha_{t} \beta_{t}+\beta_{t} S_{t}
$$

The gain $G_{t}(\varnothing)$ made by the investor via the portfolio $\varnothing$ up to time $t$ is given by

$$
G_{t}(\varnothing)=\int_{0}^{t} \alpha_{s} d \beta_{s}+\int_{0}^{t} \beta_{s} d S_{s}
$$

We say that the portfolio $\emptyset$ is self-financing if there is no fresh investment and there is no consumption. The means

$$
\begin{aligned}
& \text { that the value equals to the initial investment plus the gain: } \\
& \qquad \begin{aligned}
V_{t}(\emptyset)=x+\int_{0}^{t} \alpha_{s} d \beta_{s}+ & \int_{0}^{t} \beta_{s} d S_{s} \\
\text { (18) } & =\int_{0}^{a}\left(f(t) d B(t)+\int_{0}^{a} \beta(t) d s(t)-\frac{g(t)}{\sigma(t)}\right) d B(t)+\int_{0}^{a} \frac{g(t) B(t)}{\sigma(t) s(t)} d s(t) \\
& =\int_{0}^{a} f(t) d B(t)-\int_{0}^{a} \frac{g(t)}{\sigma(t)} d B(t)+\int_{0}^{a} \frac{g(t) B(t)}{\sigma(t) s(t)}\left(r_{t} s(t) d t+\sigma_{t} s(t) d \widetilde{N}(t)\right)
\end{aligned}
\end{aligned}
$$

Thus, we add some regularity conditions on the portfolio to exclude arbitrage opportunities.

\section{Problem Statement}

Definition 1

A self- financing strategy $\emptyset$ is said to be admissible if there exist a constant A such that $V_{t}(\varnothing) \geq-A$, a. $s . \forall t \leq T$

Definition 2

An arbitrage opportunity on the time interval $[0, T]$, is an admissible self-financing strategy $\emptyset$ such that:

1. $V_{(0)}^{\varnothing}=0$

2. $\mathbb{P}\left(V_{(T)}^{\emptyset} \geq 0\right)=1$

3. $\mathbb{P}\left(V_{(T)}^{\emptyset}>0\right)=0$

4. i.e. with zero cost today, a unit probability to have a non-negative value at time $T$ as well as a positive probability value at time $T$.

Definition 3

A contingent claim $S$, such that $\mathbb{E} \gamma^{*}\left(S^{2}\right)<\infty$ is called attainable if there exists at least one tame self-financing trading strategy $\emptyset$ such that

$V_{(T)}^{\emptyset}=S$ and we show that it is complete

\section{Hedging Electricity with the Natural Gas}

Theorem 2

If any contingent claims satisfying $\mathbb{E} \gamma^{*}\left(S^{2}\right)<\infty$ is attainable, then the associated market model is called complete.

Thus let ' $S^{\prime}$ be a contingent claim such that $\mathbb{E}_{\gamma^{*}}\left(S^{2}\right)<\infty$ as such we consider the martingale below

Proof:

$$
\begin{gathered}
f(t)=\mathbb{E}_{\gamma^{*}}\left(e^{-r T} S / w(t)\right) t \in[0, T] \\
f(t)=\mathbb{E}_{\gamma^{*}}\left(f(t)+\int_{0}^{t} g(s) d \widetilde{N}(s)\right), t \in[0, T]
\end{gathered}
$$

Since $f$ is a $\gamma^{*}-$ martingale, we have

$\mathbb{E}_{\gamma^{*}}(f(t))=f(0)$, the dynamics of the risky asset $S$ (natural gas) is given by

$$
d s(t)=r_{t} s_{t} d t+\sigma_{t} s_{t} d \widetilde{N}(t) t \in[0, T]
$$

However,

$d B(t)=r_{t} B_{t} d t, B(0)=1$ then the strategy

$\emptyset(t)=\left(\alpha_{t}, \beta_{t}\right)=\left(f(t)-\frac{g(t)}{\sigma(t)}, \frac{g(t) B(t)}{\sigma s(t)}\right)$ is the tame selffinancing strategy that replicates the contingent claim, the gain process of the strategy is 


$$
\begin{aligned}
& =\int_{0}^{a} f(t) d B(t)-\int_{0}^{a} \frac{g(t)}{\sigma(t)} d B(t)+\int_{0}^{a} \frac{g(t) B(t)}{\sigma(t) s(t)} r_{t} s(t) d t+\int_{0}^{a} \frac{g(t) B(t)}{\sigma(t) s(t)} \sigma(t) s(t) d \widetilde{N}(t) \\
& =\int_{0}^{a} f(t) d B(t)-\int_{0}^{a} \frac{g(t)}{\sigma(t)} d B(t)+\int_{0}^{a} \frac{g(t)}{\sigma(t)} d B(t)+\int_{0}^{a} g(t) B(t) d \widetilde{N}(t) \\
& =\int_{0}^{a} f(t) d B(t)+\int_{0}^{a} g(t) B(t) d \widetilde{N}(t)
\end{aligned}
$$

Recall that $f(t)=\mathbb{E}_{\gamma^{*}}\left(f(t)+\int_{0}^{t} g(s) d \widetilde{N}(t)\right)$, but $\mathbb{E}_{\gamma_{*}} f(t)=f(0)$, Thus,

Thus substituting (20) and (28) into (27), we have as follows

$$
\begin{aligned}
= & \int_{0}^{a}\left(f(0)+\int_{0}^{t} g(s) d \widetilde{N}(s)\right) d B(t)+\int_{0}^{a} g(t) B(t) d \widetilde{N}(t), \\
= & f(0) \int_{0}^{a} d B(t)+\int_{0}^{a} \int_{s}^{a} g(s) d \widetilde{N}(s) d B(t)+\int_{0}^{a} g(t) B(t) d \widetilde{N}(t) \\
= & f(0)\left(B(a)-B(0)+\int_{0}^{a} g(s) \int_{s}^{a} d B(t) d \widetilde{N}(s)+\int_{0}^{a} g(t) B(t) d \widetilde{N}(t)\right) \\
= & f(0) \int_{0}^{a} d B(t)+\int_{0}^{a} \int_{s}^{a} g(s) d \widetilde{N}(s) d B(t)+\int_{0}^{a} g(t) B(t) d \widetilde{N}(t) \\
= & f(0)(B(a)-B(0))+\int_{0}^{a} g(s)(B(a)-B(s)) d \widetilde{N}(s)+\int_{0}^{a} g(t) B(t) d \widetilde{N}(t) \\
= & f(0)(B(a)-B(0))+B(a) \int_{0}^{a} g(s) d \widetilde{N}(s) \\
= & f(0)(B(a)-B(0))+B(a) \int_{0}^{a} g(s) d \widetilde{N}(s) \\
= & f(0)(B(a)-B(0))+B(a)(f(a)-f(0)) \\
& =f(0) B(a)-f(0) B(0)+f(a) B(a)-f(0) B(a)
\end{aligned}
$$

We have that the wealth process is equals

$$
V_{(a)}^{\emptyset}=\left(f(a)-\frac{g(a)}{\sigma(t)}\right) B(a)+\left(\frac{g(a) B(a)}{\sigma(t) s(a)}\right) s(a)
$$

$=f(a) B(a) \geq 0$, thus, $\varnothing$ is a tame strategy i.e

$$
V_{(0)}^{\emptyset}=f(0) B(0)=f(0) .
$$

$\Rightarrow V_{(T)}^{\emptyset}=f(T) B(T)=S(T)$, thus

$V_{(0)}^{\emptyset}+G_{(a)}^{\emptyset}=f(0)+f(a) B(a)-f(0) B(0)=f(a) B(a)$.

Showing that $\emptyset$ is self-financing and replicates $S$ thus shows that $S$ is attainable.

\section{Results}

Dynamics of electricity price in the face of gas availability; there is a subsequent increase in power supply and increase in electricity spot-price

We have that the increase in Electricity price within the first five weeks to 20 weeks of the fourth quarter of the year, indicates the constant power supply.

\section{Discussion}

We observed that even with an increase in the price tariff, consumers, private and Government organisations will be willing to pay their light bills at regular intervals as well as the investors' interest to invest into the power sector because

\section{Conclusion}

We had developed meaningful and justified mathematical model for the pricing of electricity as well as laid down mathematical model for its risk aversion. This provides power purchasers with an effectives instruments through which they can hedge their investment in the power sector through the natural gas

\section{References}

[1] Anderson RW, Danthine J-P. 1981. Cross hedging. Journal of political Economy 89:11821196.

[2] Borenstein S. 2002.The trouble with electricity markets: Understanding California's restructuring disaster. Journal of Economic perspectives16: 191-211.

[3] Black, F. and Scholes, M. (1973). The pricing of options and corporate liabilities. Journal of Political Economy, 3.

[4] Bessemblinder H, Lemmon M. 2002. Equilibrium pricing and optimal hedging in forward markets. Journal of finance 32:654-663.

[5] Benth FE, Koekebakker S. 2008 Stochastic modelling of financial electricity contracts. Economics: 1116-1157

[6] Brennan, Michael J. and Schwartz Eduardo S (1985). Evaluating Natural Resource Investments. Journal of Business, Vol. 58, No 2, 135-153. 
[7] Carr, P.,H. Geman, D.B. Madan and M. Yor (2002). The fine structure of asset returns: an empirical investigation, Journal of Business, 75: 305-332.

[8] Cox, J.C., Ingersoll, J.E. and Ross S.A. (1985). A Theory of the term structure of Interest Rates. Econometrica 53: 385-407.

[9] Dixit, Avinvash and Pindyck, Robert S. (1994). Investment under Uncertainty. Princeton University

[10] Eydeland A,Wolynier K. 2003. Energy and power Risk management: New development in modelling, pricing and hedging John Wiley: New York.

[11] Ederington LH, Salas JM. 2008. Minimum variance hedging when spot price changes are partially predictable. Journal of Banking and finance 32:654-663.

[12] Huisaman R, Mahieu R, Schlichter F.2009. optimal peak/offpeak allocations. Energy Economics 31:169-174

[13] Karatzas, I. and Shreve, S. E. (1998). Methods of Mathematical Finance . Springer Verlag.

[14] Keppo, Jussi and Lu, Hao (2003). Real Options and a Large Producer: The Case of Electricity markets. Energy Economics,

[15] Li Y. Flynn PC. 2006. Electricity deregulation, spot price patterns and demand-side management. Energy 31: 908-922.

[16] Malliaris, A.- G. and Brock, W. (1982). Stochastic Methods in Economics and Finance. North Holland. Research, Vol. 136, No. 3: 696-706.
[17] McDonald, Robert and Siegel, Daniel (1986). The Value of Waiting to Invest. The Quarterly Journal of Economics, November, 708-727.

[18] Mun, J. (2006). Real Options Analysis: Tools and Techniques for Valuing Strategic Investments and Decisions. Hoboken, NJ: John Wiley \& Sons.

[19] Protter, P. (2004). Stochastic Integration and Differential Equations, $2^{\text {nd }}$ ed., Spring

[20] Sarkar, S., (2000). On the Investment-Uncertainty Relationship in a Real Options M1del. Journa of Economics Dynamics \& Control, 24: 219-225.

[21] Sarkar, S. (2003).The effect of mean reversion on investment under uncertainty. Journal of Economic Dynamics and Control, 28()2: 377-396

[22] sioshansi FP, Pfaffenberge. W.2006. Electricity market reform: An international perspective. Elsevier: SanDiago

[23] Xu Y. 2004. Electricity Reform in China, India and Russia. Edward Elgar: Cheltenhan

[24] Su, X. (2006). A New Approach to the Irreversible Investment Problem, Technical report, Bonn Econ Paper No. 21/2006.

[25] Woo, C.K., King, M., Tishler A., Chow, L.C.H., 2006c. Costs of electricity deregulation. Energy, 31, 747-768.

[26] Woo C.K., L loyd D. Tishler A. 2003. Electricity market reform failures: UK, Norway. 